\title{
Measuring Flow Velocity and Gas Concentration in Binary Gas Mixtures Using Thermal Sensors
}

\author{
Hepp, Christoph ${ }^{1}$; Krogmann, Florian ${ }^{1}$; Polak, Jiri ${ }^{1}$; Lehmann, Mirko ${ }^{1}$; Urban, Gerald ${ }^{2}$ \\ ${ }^{1}$ Innovative Sensor Technology AG ${ }^{2}$ IMTEK, University of Freiburg \\ Industriestrasse 2 \\ 9630 Wattwil \\ Switzerland \\ Georges-Köhler-Allee 103 \\ 79100 Freiburg \\ Germany
}

\begin{abstract}
Introduction
Gas sensors are used in many different applications, for example in air conditioning technology or fire detectors. Moreover gas sensors are playing more and more an important role in process control of biogas plants. The actual high price of fossil fuels show that it is wise to establish renewable energies beside the ecological point of view. A sensor element measuring both flow velocity and gas concentration of methane - integrated on one single chip - is a huge improvement of process reliability in biogas plants. A precise control of the fermentation process can be achieved by mounting such a sensor close to the fermenter. A further feature of the sensor will be the exact calculation of the fuel value from flow speed and gas concentration.
\end{abstract}

Optical systems based on the spectroscopy and thermal conductivity sensors are state of the art by the analysis of biogas. Spectrometers show a low cross contamination by the detection of methane to other gases, enabling a high accuracy in the measurement of gas concentration. One disadvantage of these systems is the high price; in contrast to thermal conductivity sensors which are easy to fabricate and in this way obtain a low price. Further advantages are the robustness and the long-time cycle of these sensor systems. However the flow rate has to be kept constant to avoid measuring errors.

Flow sensors based on thermal principles offer many advantages compared to other flow sensors such as wheel blower or variable area flow meter. Thermal flow meters are characterised by an increased reliability and durability due to the absence of moving parts. However, these sensors do not work medium independently and have to be calibrated on the specific gas applied in [1]. The calibration process is costly, time-consuming and very complicated if the flow sensor is applied in a gas mixture where the gas concentration is fluctuating by time. This drawback can be overcome by measuring the thermal gas properties of the fluid simultaneously to the flow measurement [2, 3].

Constant thermal anemometry is established to measure flow measurement with thermal devices. For this purpose a hot wire is exposed to a moving fluid. The heat transfer increases with a rise in flow velocity. The flow velocity is calculated from electrical power needed to heat the wire to a given temperature above fluid temperature. Another possibility measuring flow speed is the calorimeter principle. By this mode the sensor consists of an up- and downstream temperature sensor and a heater in between. The fluid is constantly heated up and the temperature difference between up- and downstream temperature sensor is detected to calculate flow speed [4].

AC-excitation of the heater - located between different temperature sensors - allows additional information like phase shift and amplitude of the temperature sensors. This enables the measurement of thermal gas properties in no flow condition [5]. Flow speed is detectable, for example, with a DC-heating of the heater and measuring the temperature difference between up- and downstream temperature sensor [6].

A variation of the thermal gas properties by modifying the gas concentration has an influence on the amplitude temperature difference between up- and downstream temperature sensor signals and yields in an uncertainty of flow speed measurements. The challenge during this procedure is the extraction of the flow velocity independent of the thermal gas properties.

In the following a sensor design and an characterisation with $A C$ heating is presented in order to investigate the influence in gas concentration and flow speed change on thermal flow sensor. 


\section{Concept and Design}

The chip design is based on the structure of an unsymmetrical resistor array to detect the temperature distribution. The sensor is equipped with an up- and downstream platinum resistance used as temperature sensor and a further platinum resistance used as heater, located in between. Figure 1 shows a detailed view of the sensor.

An unsymmetrical 1D-array was selected in order to optimise the measurement of a change in temperature distribution due to variation in flow speed or gas concentration. The separation distance between upstream temperature sensor and heater is designed with $435 \mu \mathrm{m}$. In contrast, the separation distance between downstream temperature sensor and heater is fabricated with $190 \mu \mathrm{m}$.

The temperature sensors are designed with a resistance of $750 \Omega$ in order to achieve a high temperature resolution with a minimum of $0.1 \mathrm{~K}$. These chosen resistance values provide the required sensitivity to detect tiny changes in the fluid temperature (due to a variation in flow speed or gas composition). The array is located on a thin membrane to increase the sensors sensitivity and furthermore to minimize the disturbing thermal conductivity through a substrate. As membrane material a polymer is chosen to improve the thermal isolation between heater and temperature sensors. Foturan - a photosensitive glass - is used as substrate material enabling a robust sensor handling. A further advantage in the combination of these materials is the very low eduction of thermal power via membrane and phase shift in comparison to other membrane and substrate materials.

\section{Fabrication process}

The fabrication process is shown in Fig. 2. Foturan can be patterned by UV-expose and annealing. Crystals of lithium are formed through this procedure, which can be etched with hydrofluoric acid [7]. Such a Foturan wafer is used as substrate material. Polyimide - a high temperature polymer - is chosen as polymer and spin coated on the front side of the wafer. This coating step forms a film thickness of around $6 \mu \mathrm{m}$. An annealing step causes the cross linking and performs a high temperature stability of the Polyimide. It follows the deposition of platinum by a PVD process and structuring by dry etching (on the Polyimide coated wafer side). Again, Polyimide is coated on top and used as sensor passivation. The contact pads are opened with a wet etch step. An annealing step is performed as well for cross linking. The membrane is established by a wet etch process. The crystallized parts of Foturan removed with hydrofluoric acid.

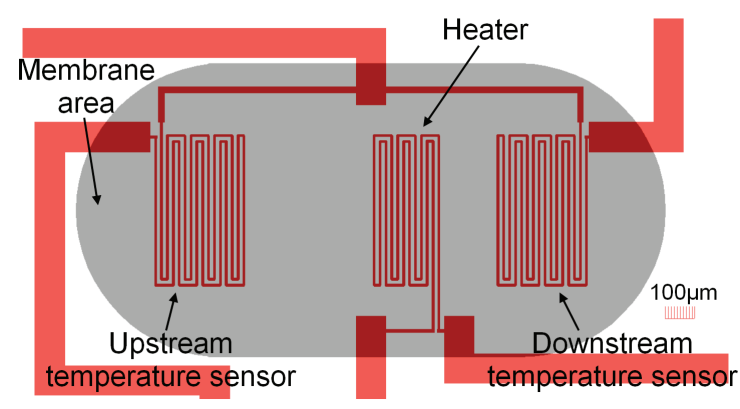

Fig.1: View of the sensor

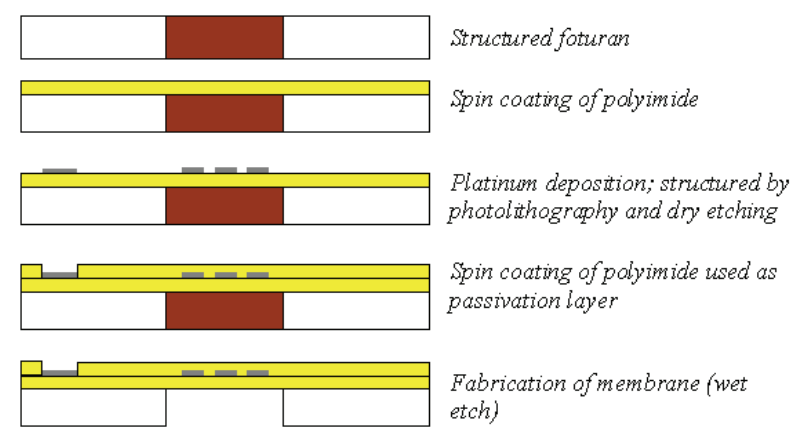

Fig. 2: Fabrication Process

\section{Measurement setup and results}

Experiment 1: Change in gas concentration

Primarily the sensor is characterised in a no flowing binary gas mixture consisting of carbon dioxide and methane. The sensor was mounted in a gas chamber with temperature control. Carbon dioxide and methane was mixed by two different mass flow controllers (MFC) to obtain the desired gas concentration. The total flow was adjusted 500mililiter per min and kept constant in order to flush the chamber every 3 minutes. Therefore no flow velocity was practically measurable at the sensor. The variation of the MFC's volume flow changes the gas concentration. The binary gas mixture was set up stepwise to a concentration of $0 \%, 40 \%, 50 \%, 70 \%$ and $100 \%$ of methane. The change of gas species from carbon dioxide to methane implies a variation of the gas' thermal conductivity from $\lambda=16.5 \mathrm{~mW} / \mathrm{mK}$ to $\lambda=33.9 \mathrm{~mW} / \mathrm{mK}$. In order to investigate the hysteresis of the system the measurement was carried out the way round as a gas concentration of $100 \%$ methane was reached. In this experiment the relative humidity of the gas mixture was zero per cent.

In a further experiment the gas composition's humidity was varied to examine the effect on amplitude and phase shift. For this purpose the gas was conducted through a humidifier before reaching the gas chamber. 
The time dependent modulation of the heating power was created with function generator. As excitation a sinus signal with a maximum power of $17 \mathrm{~mW}$ and a frequency of $1 \mathrm{~Hz}$ was applied on the heater.

For the data acquisition a voltage divider was made from a series resistor and the temperature sensor located on the chip. The voltage to be measured was referred on the temperature sensor. The periodic voltage variation on the temperature sensors - caused through the time depending heating on the heater - is acquired with a DAQ-card from National Instruments. A LabVIEW programme was written for the evaluation process. The programme determines through a fast fourier transformation (FFT) the amplitude value and the phase shift between the excitation signal of the heater and the down- and upstream temperature sensors.

The results of this measurements show that amplitude is decreasing with an increase of thermal conductivity; illustrated in Fig. 3 and 4. The amplitude value is reduced from $5.7 \mathrm{mV}$ to $3.6 \mathrm{mV}$ on the low separation distance. At the low separation distance the amplitude is lower compared to the high separation distance and achieves a value of $2.25 \mathrm{mV}$ and is further reduced with an increase of thermal conductivity to $1.6 \mathrm{mV}$. An amplitude change of $5 \mathrm{mV}$ corresponds to a temperature difference of $20 \mathrm{~K}$.

The terms between heater and temperature sensors is reduced by a change in the gas composition from carbon dioxide to methane as shown in Fig. 5. A phase shift difference is the result of the different separation distance. In this experiment the phase shift of 1 rad corresponds to a time duration of $159.1 \mathrm{~ms}$. A change in gas humidity can be detected in a variation of amplitude and phase shift. The amplitude is decreasing around $3 \%$ independent of the separation distance due to the change in humidity. The change in relative humidity leads to a significant increase of the time duration (see Fig. 4, 5, 6). The hysteresis of the system is negligible - for both amplitude and phase shift.

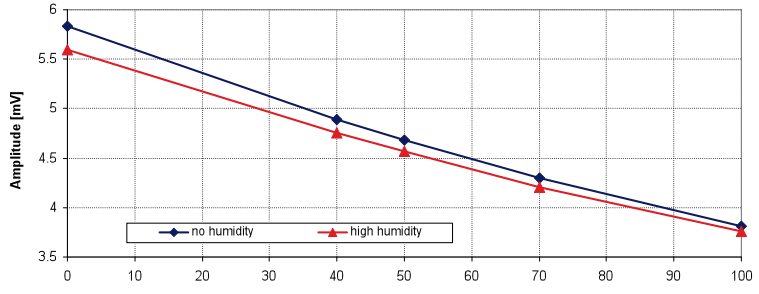

CH4 concentration in gas mixture of $\mathrm{CH} 4$ and $\mathrm{CO}[\%]$

Fig. 3: Amplitude variation of upstream temperature sensor as function of methane gas concentration.

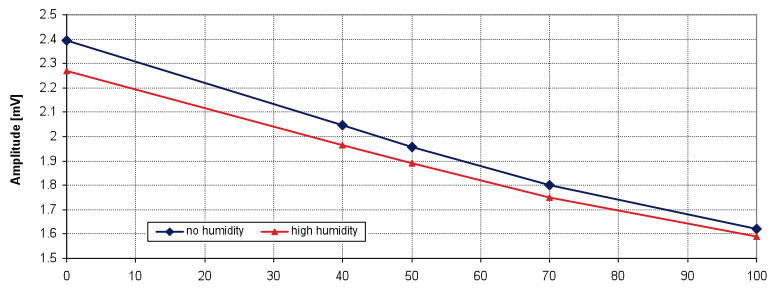

CH4 concentration in gas mixture of $\mathrm{CH} 4$ and $\mathrm{CO} 2 \%$

Fig. 4: Amplitude variation of downstream temperature sensor as function of methane gas concentration.

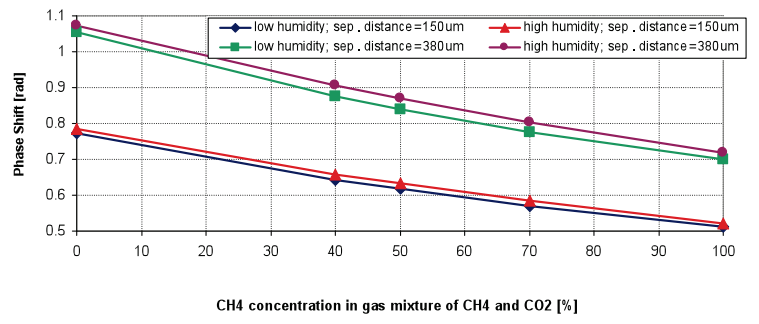

Fig. 5: Comparison of phase shift between heater and temperature sensors (up- and downstream) as function of methane gas concentration.

\section{Experiment 2: Change in flow velocity}

Another experiment was carried out in order to investigate the variation of amplitude and phase shift due to the change in flow speed. Therefore the sensor was mounted in the middle of a round flow channel with a diameter of $6 \mathrm{~mm}$, active sensor side facing parallel to flow direction. At the control unit of the MFC, the gas flow was increased stepwise from zero sccm to a maximum value of $1400 \mathrm{sccm}$ and again stepwise reduced to zero sccm. The volume flow of $1400 \mathrm{sccm}$ corresponds to a calculated flow speed of $0.9 \mathrm{~m} / \mathrm{s}$. Dry air was used as flowing gas medium for this measurement. 
The modulation of the heating power was again performed with a function generator. The maximum heating power was reduced to $11 \mathrm{~mW}$. The other attitudes remained unchanged. The data acquisition and evaluation was accomplished as described in the previous section.

The results demonstrate that phase shift and amplitude is affected by flow speed. As illustrated in Fig. 6 the amplitude of the upstream temperature sensor is decreasing with an increase in flow velocity; the amplitude of the downstream is increasing until approximately $700 \mathrm{sccm}$; afterwards it is slightly decreasing. Phase shift is decreasing with an increase of flow velocity as illustrate in Fig. 7. A higher separation distance causes in a higher time duration compared to the lower distance. The gradient of the upstream temperature sensor curve is almost constant within the entire flow range. In contrast to the gradient of the downstream temperature sensor curve, the gradient is getting lower around $800 \mathrm{sccm}$. This results in an unconstant difference in phase shift between up- and downstream temperature sensor. The difference is nearly constant up to a flow velocity of $800 \mathrm{sccm}$ (corresponds to $0.5 \mathrm{~m} / \mathrm{s}$ ). Afterwards it is decreasing rapidly as Fig. 7 demonstrates.

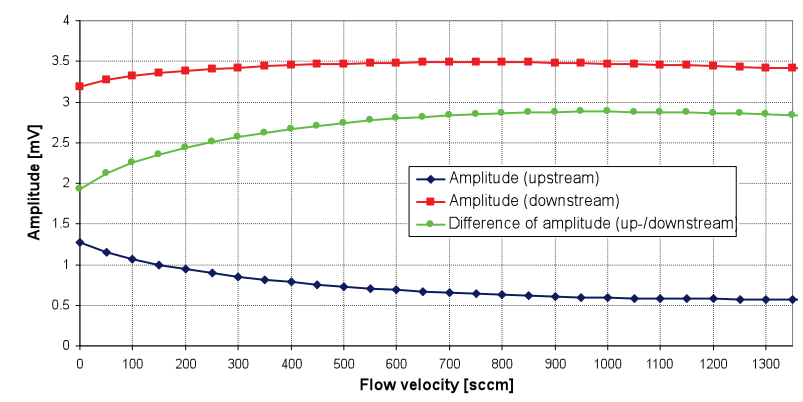

Fig. 6. Amplitude variation of downstream and upstream temperature sensor as function of flow velocity.

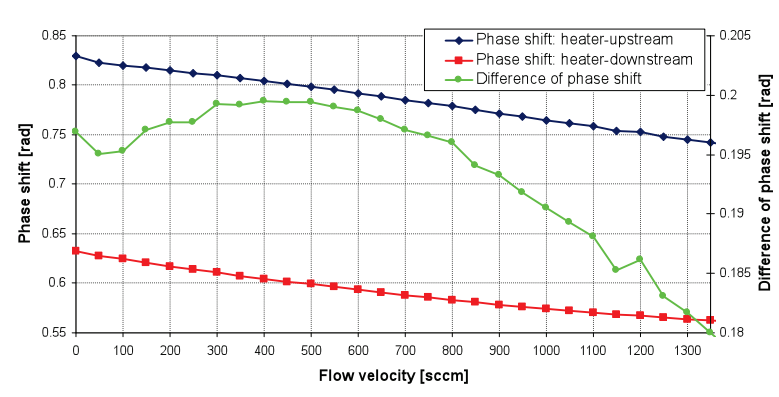

Fig. 7: Variation of phase shift of upstream and downstream temperature sensor to heater as function of flow velocity.

\section{Conclusion and Outlook:}

According to these results it should be possible to determine gas concentration and flow speed within a certain flow range. Amplitude depends on flow velocity and gas concentration as well the phase shift between excitation and the different temperature sensors. However, the difference in phase shift between upstream and downstream temperature sensor is not affected by flow speed within a certain range. This flow range can be used to determine the gas concentration by the difference in phase shift. Afterwards the accurate flow velocity can be calculated by the amplitude. The flatness of the phase shift is already sufficient to calculate the thermal conductivity with a measuring error of $15 \%$ (comparison of the gas' thermal conductivity used in Fig. 3 and Fig. 6).

At this point the following application is conceivable. The sensor is mounted in a flow channel where flow speed is under $800 \mathrm{sccm}$. In this range the difference in phase shift is not affected by flow velocity and varies only due to a change in gas composition. Due to this variation the gas composition can be achieved and, for example, a warning can be displayed when the gas concentration is not within the tolerance range. Afterwards the accurate flow velocity can be determined.

\section{References:}

[1] T. Lammerink, F. Dijkstra, Z. Houkes, J. van Kuijk, "Intelligent gas-mixture flow sensor", Sensors and Actuators A: Physical, Vol. 47, No. 1, pp. 380-384, 1995.

[2] H. Ernst, A. Jachimowicz, G. Urban, "Dynamic thermal sensor-principles in MEMS for fluid characterization", IEEE Sensors Journal, Vol. 1, No. 4, pp. 361-367, 2001.

[3] A.S. Cubukcu, G. Urban, "Simulation and fabrication of a 2D-flow sensor for simultaneous fluid characterization", Proceedings of the Eurosensors XXIII Conference, Procedia Chemistry, Lausanne, Switzerland, Sept. 6-9, 2009, pp. 887-890.

[4] M. Ashauer, H. Glosch, F. Hedrich, N. Hey, H. Sandmaier, W. Lang, "Thermal flow sensor for liquids and gases based on the combinations of two principles", Sensors and Actuators A: Physical, Vol. 73, pp. 7-13, 1998.

[5] J. Kuntner, F. Kohl, B. Jakoby, „Simultaneous thermal conductivity and diffusivity sensing in liquids using a micromachined device", Sensors and Actuators A: Physical, Vol. 130-131, pp. 62-67, 2006. 
[6] M. Elwenspoek, "Thermal flow micro sensors", Proceedings of the International Semiconductor Conference, Vol. 2, Sinaia, Romania, Oct. 5-9, 1999, pp. 423-435.

[7] Mikroglas chemtech GmbH, Datasheet, www.mikroglas.com, 2010. 